\title{
Development of a Diet Management Scale for Pediatric Patients with Citrin Deficiency
}

\author{
Ling Yan ${ }^{1}$, Yuanzong Song ${ }^{2}$, Meng Zhang ${ }^{1}$, Jianwu Qiu ${ }^{2}$, Chong Jiang ${ }^{3}$, Qingran Lin ${ }^{1,}$ * \\ ${ }^{1}$ Department of Nursing, The First Affiliated Hospital of Jinan University, Guangzhou, China \\ ${ }^{2}$ Department of Pediatrics, The First Affiliated Hospital of Jinan University, Guangzhou, China \\ ${ }^{3}$ Department of Stomatologist, Nanfang Hospital, Southern Medical University, Guangzhou, China
}

Email address:

qingranlin@126.com (Qingran Lin)

${ }^{*}$ Corresponding author

\section{To cite this article:}

Ling Yan, Yuanzong Song, Meng Zhang, Jianwu Qiu, Chong Jiang, Qingran Lin. Development of a Diet Management Scale for Pediatric Patients with Citrin Deficiency. European Journal of Preventive Medicine. Vol. 8, No. 2, 2020, pp. 16-23. doi: 10.11648/j.ejpm.20200802.12

Received: March 15, 2020; Accepted: March 31, 2020; Published: April 23, 2020

\begin{abstract}
Objective: This study aimed to develop a scale to assess the caregiversability to manage the dietary needs of pediatric patients with citrin deficiency (CD). Methods: 24 experts from 11 hospitals in mainland China participated in the Delphi research. Item analysis was performed on 61 caregivers of CD patients who were enrolled in the testing of the draft scale, and 153 caregivers attended the validity and reliability testing. The initial scale was made according to the suggestions by the 24 experts. Retest reliability method and Cronbach's $\alpha$ coefficient method were used to evaluate the external and internal reliability of the scale, respectively; the scale validity was evaluated by content validity and construct validity. Results: A consensus was reached by 24 experts after two rounds of investigation, and a preliminary draft of the scale was formed consisting of three dimensions: Special dietary knowledge, Healthy dietary attitude and Dietary behavior. The expert authority coefficient was 0.887 , and the Kendall coefficient was 0.201. The final scale Cronbach's alpha coefficient $(\alpha=0.713)$ showed good internal consistency and balance stability. The retest reliability coefficient of the scale was 0.975 , indicating good external stability. The total content validity index (S-CVI) was 0.981 , and the content validity index (I-CVI) of each item varied between 0.913 and 1.0. On confirmatory factor analysis, the indicators CMIN/DF, RESAE, PNFI, NFI, TLI, IFI and CFI for the construct validity of the scale were $1.191,0.053,0.628,0.796,0.940,0.954$, and 0.951 , respectively. Conclusion: The scale developed in this paper exhibited promising reliability and validity for the clinicians to evaluate the dietary management ability of the caregivers of pediatric CD patients.
\end{abstract}

Keywords: Scale, Citrin Deficiency, Diet Manage, Caregiver

\section{Introduction}

Citrin deficiency (CD) is a genetic metabolic disease caused by biallelic mutations of SLC25A13 gene [1]. This disease has three clinical phenotypes: Neonatal Intrahepatic Cholestasis caused by Citrin Deficiency (NICCD) [1], Failure to Thrive and Dyslipidemia caused by Citrin Deficiency (FTTDCD) [2], and adult-onset citrullinemia type II (CTLN2) [1]. Previous studies have shown that excessive carbohydrate intake is harmful to $\mathrm{CD}$ patients [3-5]. Management of CD requires adherence to a low-carbohydrate while high-protein and high-lipid dietary therapy [6-8]. Growth impairment may occur in children if the diet is not properly managed [9].
Dietary management is the major therapeutic measure for $\mathrm{CD}$, an inbor error of metabolism arising from biallelic mutations of the gene SLC25A13. The effect of dietary management in pediatric patients with $\mathrm{CD}$ depends on the management ability of their caregivers; however, to the best of our knowledge, there is currently no specific scale to evaluate such a ability. To address this issue, this study aimed to develop a diet management scale for the caregivers of pediatric patients with $\mathrm{CD}$. We hope that through this scale, caregivers will be able to manage the diet of patients, improve the management abilities of caregivers, and improve the quality of life of children. 


\section{Methods}

\subsection{Study Design}

After conducting a thorough literature review to form a pool of potential questions for the scale, experts screened the pool of entries using the Delphi method. Then exploratory factor analysis was done, and questions were divided into 4 dimensions. Finally analysis on the reliability and validity of the scale was completed.

\subsection{Construction of the Scale Items Pool}

Through the literature review, related field expert consultation and group discussion, 31 items were extracted. Among them, 8 items involved the dietary knowledge dimension, 6 items the dietary belief dimension, and 17 items in the eating behavior dimension.

\subsection{Delphi Expert Consultation}

\subsubsection{Investigate Experts Into the Standard}

Qualifications for the Delphi experts included: 1) Working in a tertiary hospital or college; 2) Acquired intermediate titles; 3) Genetics research in the field of hepatology; 4) Researcher in the field of nutrition; 5) Specialist nurse; 6) Agreement to participate in the research study.

\subsubsection{Delphi Expert Questionnaire Standard}

The importance of each item on the scale is represented by the mean of the expert score ( $\geq 4$ is important) [10]. The degree of expert authority is represented by the expert authority coefficient $(\mathrm{Cr})(\geq 0.7$ is considered good) and the positive degree of an expert is represented by questionnaire recovery rate and expert opinion. Coefficient of variation $(\mathrm{CV})>0.25$ indicates poor consistency of expert scores. Expert opinion dispersion degree is represented by Kendall's $\mathrm{W}(0-1)$. A larger value indicates better coordination.

\subsubsection{Delphi Questionnaire Items Deletion Criteria}

Items that normally meet any of the following requirements are deleted, depending on the actual situation [11]. This study used: 1) Coefficient of variation $>0.25 ; 2$ ) Mean of expert ratings $<4$; 3) Outer number of items $<20 \%$.

\subsection{Formation of the Citrin Defective Diet Management Scale}

Literature shows that the sample size can be 3 times the number of items [12]. In this research, caregivers of children diagnosed with $\mathrm{CD}$ were the test subjects. The investigator explained to each subject the purpose and method of filling out the questionnaire on the "questionnaire star" APP. Items were scored according to the following criteria: Dimensions 1 scoring criteria: option plus one point, error option minus 0.5 points; Dimension 2 scoring criteria: items were highly dissatisfied, disapproved, uncertain, agree, and strongly endorsed according to the Likert 5 rating; Dimension 3 items were assigned 1-5 points for never, occasionally, average, often and always according to the Likert 5 rating scale. The scores of the scales were ranked from high to low, and then the independent sample $t$ test was used to compare the scores of the items in the top $27 \%$ (high group) and the last $27 \%$ (low group) items, with the retention decision being $(\mathrm{CR})$ value $>3(P<0.05)$ [13]. Exploratory factor analysis was then completed to determine the dimension of the scale based on the results of exploratory factor analysis, to optimize the scale structure. The Kaiser-Meyer Olkin (KMO value) and Bartlett sphere test (KMO value $>0.7)$ and Bartlett's spherical test were statistically significant $(P<0.05)$, indicating that items were suitable for the factor analysis [13]. Principal component analysis, skew rotation axis, maximum variation method are used to automatically extract the factor root $(>1)$ and factor load $(>0.4)$ for the factor matrix. A steep slope map helped determine the number of factors, delete multiple loads and the load values that were close. A factor of less than 3 items, after each factor analysis, necessitated re-assessment of the item $d$ to determine if it should be deleted. Each time one item was deleted, the factor analysis was performed again, and the dimension of the item was finally adjusted. When the total variation (the extracted factors combined to explain all variables) reached $50 \%$, the results were acceptable, and if it reached $60 \%$, the factors retained after extraction were ideal [13].

\subsection{Instrument}

This data was complied with Excel 21.0 and SPSS21.0 for data analysis. Communication with subjects was completed using E-mail. The caregiver questionnaire was distributed through questionnaire software.

\subsection{Sampling}

\subsubsection{Expert Source}

The 24 experts who accepted participation in this study the inquiry were from 10 provinces, autonomous regions and municipalities in China. All experts were from tertiary hospitals or higher education institutions. In addition, one expert as a specialist nurse and the other 23 experts were either senior nursing staff or senior faculty.

\subsubsection{Source of Caregivers for Children with Citrin Deficiency}

The caregivers who participated in the study were caregivers of children diagnosed with CD. They were recruited from the pediatric outpatient department of the First Affiliated Hospital of Jinan University in China between January 2015 to September 2019.

\subsection{Ethical Considerations}

This study was approved by the Ethics Association of the First Affiliated Hospital of Jinan University. All questionnaires were anonymous and all the caregivers in the survey had signed informed consent forms. 


\section{Results}

\subsection{Delphi Expert Consultation Questionnaire Results}

\subsubsection{Expert Enthusiasm}

Of the 24 experts interviewed in this study, one was from a college, 23 from hospitals, 2 nursing experts and 22 clinical medical experts. Expert research interests included gastroenterology, nutrition, hepatology, and chronic disease management. For the 2 rounds of Delphi inquiry 24 packets were distributed. In the first round the effective recovery rate of $100 \%$, and the expert opinion rate was $58.3 \%$. In the second round 23 experts received materials and the effective recovery rate was $95.8 \%$ and the expert opinion rate was
$52.2 \%$.

\subsubsection{Expert Authority}

Result of the Expert authority are shown in Table 1.

Table 1. Expert authority.

\begin{tabular}{llll}
\hline Stage & $\begin{array}{l}\text { Judgment } \\
\text { coefficient }(\text { Ca) }\end{array}$ & $\begin{array}{l}\text { Familiarity } \\
\text { (Cs) }\end{array}$ & $\begin{array}{l}\text { Authority } \\
\text { cofficient (Cr) }\end{array}$ \\
\hline Round 1 & 0.942 & 0.858 & 0.900 \\
Round 2 & 0.948 & 0.826 & 0.887 \\
\hline
\end{tabular}

\subsubsection{Degree of Coordination of Expert Opinions}

Result of the degree of coordination of expert opinions are shown in Table 2 .

Table 2. Degree of coordination of expert opinions.

\begin{tabular}{|c|c|c|c|c|c|}
\hline \multicolumn{3}{|c|}{ Round 1} & & \multicolumn{2}{|l|}{ Round 2} \\
\hline & Kendall's W & $P$ & & Kendall's W & $P$ \\
\hline Dimension & 0.187 & $P<0.05$ & Dimension & 0.201 & $P<0.05$ \\
\hline Items & 0.212 & $P<0.00$ & Items & 0.30 & $P<0.00$ \\
\hline
\end{tabular}

\subsubsection{Second Round of Expert Inquiry Results}

Result of the expert inquiry in second round are shown in Table 3.

Table 3. Expert inquiry in second round.

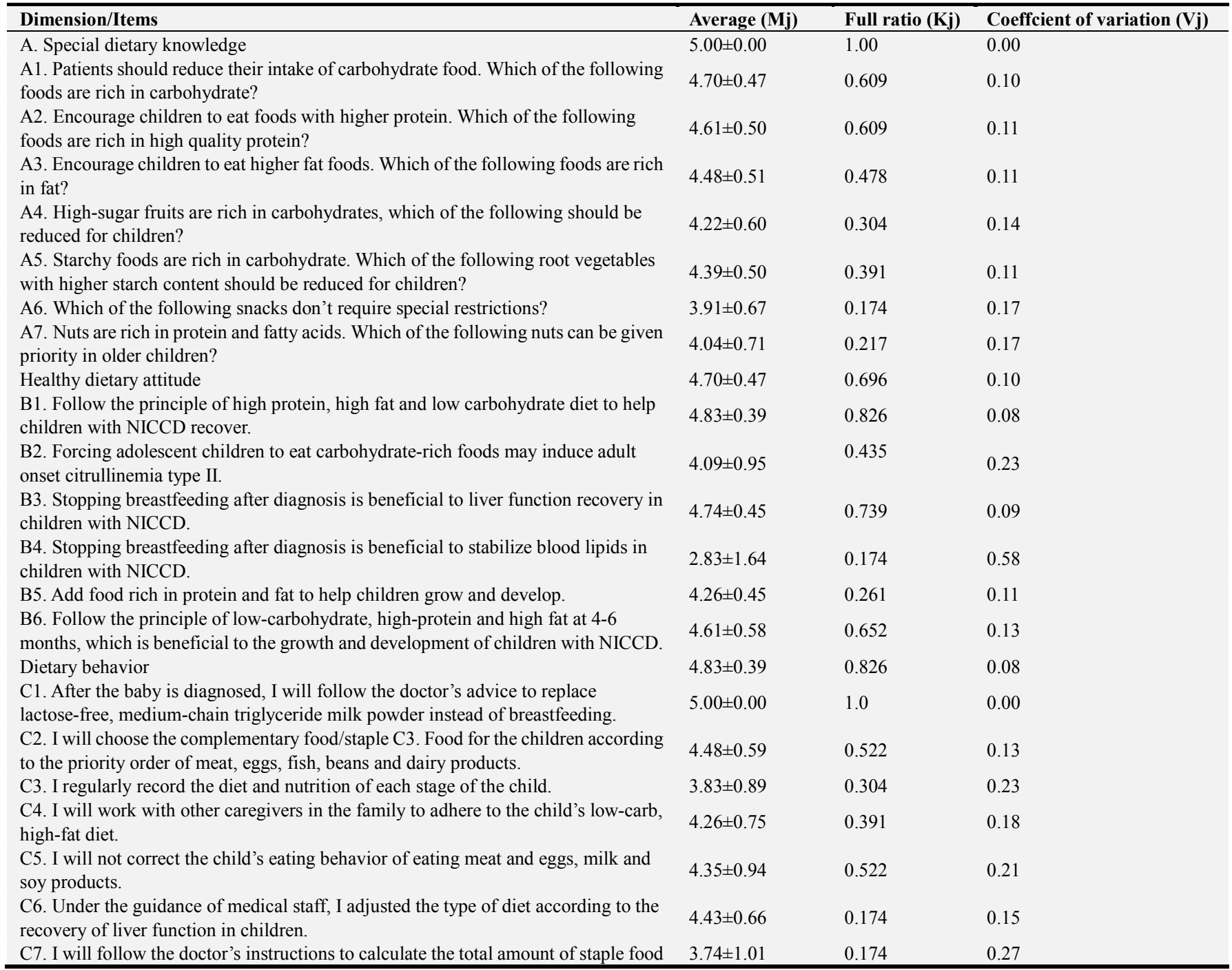




\begin{tabular}{|c|c|c|c|}
\hline Dimension/Items & Average (Mj) & Full ratio $(\mathbf{K j})$ & Coeffcient of variation $(\mathrm{Vj})$ \\
\hline \multicolumn{4}{|l|}{ that the child needs to add. } \\
\hline C8. I will adjust the child's diet according to the nutritional status of the child. & $4.00 \pm 0.95$ & 0.304 & 0.24 \\
\hline $\begin{array}{l}\text { C9. Children with this disease have special dietary preferences. I will not force } \\
\text { children to eat foods rich incarbohydrates, noodles, etc. }\end{array}$ & $4.70 \pm 0.56$ & 0.739 & 0.12 \\
\hline C10. I will not test the tolerance of children ro rice noodles. & $3.65 \pm 0.83$ & 0.174 & 0.23 \\
\hline $\begin{array}{l}\text { C11. I will ask the medical staff about my doubts } \\
\text { about diet management. }\end{array}$ & $4.52 \pm 0.79$ & 0.652 & 0.17 \\
\hline $\begin{array}{l}\text { C12. If the child enters school, I will communicate with the teacher and tell the } \\
\text { principle of eating. }\end{array}$ & $4.57 \pm 0.73$ & 0.696 & 0.16 \\
\hline $\begin{array}{l}\text { C13. If the child enters school, I will prepare a high-protein, high-fat, } \\
\text { low-carbohydrate meal for the child to take to school. }\end{array}$ & $3.48 \pm 1.04$ & 0.217 & 0.30 \\
\hline $\begin{array}{l}\text { C14. If the child enters school, I will train the child's special diet knowledge and } \\
\text { self-catering management skills. }\end{array}$ & $4.70 \pm 0.64$ & 0.783 & 0.14 \\
\hline C15. I will actively learn about Citrin deficiency disease nutrition management. & $4.61 \pm 0.50$ & 0.609 & 0.11 \\
\hline $\begin{array}{l}\text { C16. If the child enters school, I will tell the child about the toxicity of alcohol to the } \\
\text { liver. }\end{array}$ & $3.70 \pm 1.11$ & 0.348 & 0.30 \\
\hline $\begin{array}{l}\text { C17. If the child's disease is improved, I will try to add lactose-containing dairy } \\
\text { products to the child. }\end{array}$ & $3.13 \pm 1.10$ & 0.130 & 0.35 \\
\hline $\begin{array}{l}\text { C18. I will pay attention to whether the food ingredients are harmful to the health of } \\
\text { the children. }\end{array}$ & $4.26 \pm 0.81$ & 0.478 & 0.19 \\
\hline
\end{tabular}

\subsection{The Results of Scale Entry Screening}

\subsubsection{Item Analysis Result}

A total of 80 questionnaire were distributed to caregivers of children with $\mathrm{CD}$, and the 61 questionnaires were effectively recovered. The effective recovery rate was $76.3 \%$ in this study.

The frequency analysis of the answers to the 26 entries of the initial scale shows that the selection rate of the other items is less than $80 \%$ except that the selection rate of the original item $\mathrm{C} 14$ is $94 \%$ ( $>80 \%)$. Entry C14 was deleted and the remaining entries were retained for further analysis.

\subsubsection{Cronbach's $\alpha$ Coefficient Result}

The Cronbach's $\alpha$ coefficient results for each dimension on the first draft of the scale are shown in Table 4. The Cronbach's $\alpha$ coefficient of the corresponding dimension and totals after deleting the entry is shown in Table 5. The result shows that after deleting the item A3, the Cronbach's $\alpha$ coefficient of dimension one rises to 0.767 , which is higher than the original 0.740 , so A3 was deleted. After deleting the item A8, the dimension Cronbach's $\alpha$ coefficient of one rises to 0.745 , which is higher than the original 0.733 . After deleting the item B13, the Cronbach's $\alpha$ coefficient of dimension two rises to 0.903 , which is higher than the original 0.725 . After deleting the item $\mathrm{C} 14$, the Cronbach's $\alpha$ coefficient rose to 0.767 , higher than the original 0.733 , and was deleted. After deleting the item $\mathrm{C} 18$, the Cronbach's $\alpha$ coefficient of dimension three rose to 0.768 , which was higher than the original 0.731 . After deleting the item $\mathrm{C} 19$, in dimension three, the Cronbach's $\alpha$ coefficient rises to 0.770 , which is higher than the original 0.732 . After deleting the item $\mathrm{C} 21$, the Cronbach's $\alpha$ coefficient of dimension three rises to 0.777 , which is higher than the original 0.736 . The specific values are shown in Table 5.

Table 4. Cronbach's a coefficient results on the first draft of the scale.

\begin{tabular}{lll}
\hline Dimension & Number of items & Cronbach's $\boldsymbol{\alpha}$ coefficient \\
\hline Dimension 1 & 8 & 0.733 \\
Dimension 2 & 5 & 0.871 \\
Dimension 3 & 13 & 0.779 \\
\hline
\end{tabular}

Dimension 1 represents Special dietary knowledge; Dimension 2 represents Healthy dietary attitude; Dimension 3 represents Dietary behavior.

Table 5. Result of the Item filtering.

\begin{tabular}{|c|c|c|c|c|c|}
\hline Items & Overall Scale $(0.786)$ & Dimension $1(0.733)$ & Dimension 2 (0.871) & Dimension 3 (0.779) & Retention \\
\hline $\mathrm{A} 1$ & .770 & .702 & & & Reserved \\
\hline $\mathrm{A} 2$ & .767 & .683 & & & Reserved \\
\hline A3 & .798 & .750 & & & Delete \\
\hline A4 & .764 & .665 & & & Reserved \\
\hline A5 & .776 & .695 & & & Reserved \\
\hline A6 & .788 & .736 & & & Delete \\
\hline A7 & .772 & .661 & & & Reserved \\
\hline A8 & .788 & .742 & & & Delete \\
\hline B9 & .778 & & .834 & & Reserved \\
\hline $\mathrm{B} 10$ & .779 & & .840 & & Reserved \\
\hline B11 & .776 & & .823 & & Reserved \\
\hline B12 & .775 & & .807 & & Reserved \\
\hline B13 & .774 & & .921 & & Delete \\
\hline $\mathrm{C} 14$ & .787 & & & .785 & Delete \\
\hline $\mathrm{C} 15$ & .780 & & & .759 & Reserved \\
\hline
\end{tabular}




\begin{tabular}{|c|c|c|c|c|c|}
\hline Items & Overall Scale (0.786) & Dimension 1 (0.733) & Dimension 2 (0.871) & Dimension 3 (0.779) & Retention \\
\hline C16 & .773 & & & .779 & Reserved \\
\hline $\mathrm{C} 17$ & .782 & & & .759 & Reserved \\
\hline $\mathrm{C} 18$ & .784 & & & .778 & Reserved \\
\hline C19 & .785 & & & .777 & Reserved \\
\hline $\mathrm{C} 20$ & .779 & & & .743 & Reserved \\
\hline $\mathrm{C} 21$ & .791 & & & .789 & Delete \\
\hline $\mathrm{C} 22$ & .783 & & & .746 & Reserved \\
\hline $\mathrm{C} 23$ & .779 & & & .744 & Reserved \\
\hline $\mathrm{C} 24$ & .776 & & & .747 & Reserved \\
\hline $\mathrm{C} 25$ & .776 & & & .747 & Reserved \\
\hline $\mathrm{C} 26$ & .778 & & & .745 & Reserved \\
\hline
\end{tabular}

(Consider deleting the underlined items in the Table5).

\subsection{Final Scale Reliability and Validity Test}

\subsubsection{General Situation of the Research Object}

A total of 135 questionnaire were distributed to caregivers of children with $\mathrm{CD}$, and the 133 questionnaires were effectively recovered. The effective recovery rate was $98.52 \%$ in this study.

\subsubsection{Exploratory Factor Analysis Results}

The KMO value is 0.727 , and the Bartlett spherical test $\chi^{2}$ $=636.181, P<0.00$, suitable for line factor analysis. Principal component analysis and skewed axis automatically propose 4 common factors (feature value $>1$ ). In Figure 1, the gravel map slows down after the fifth factor.

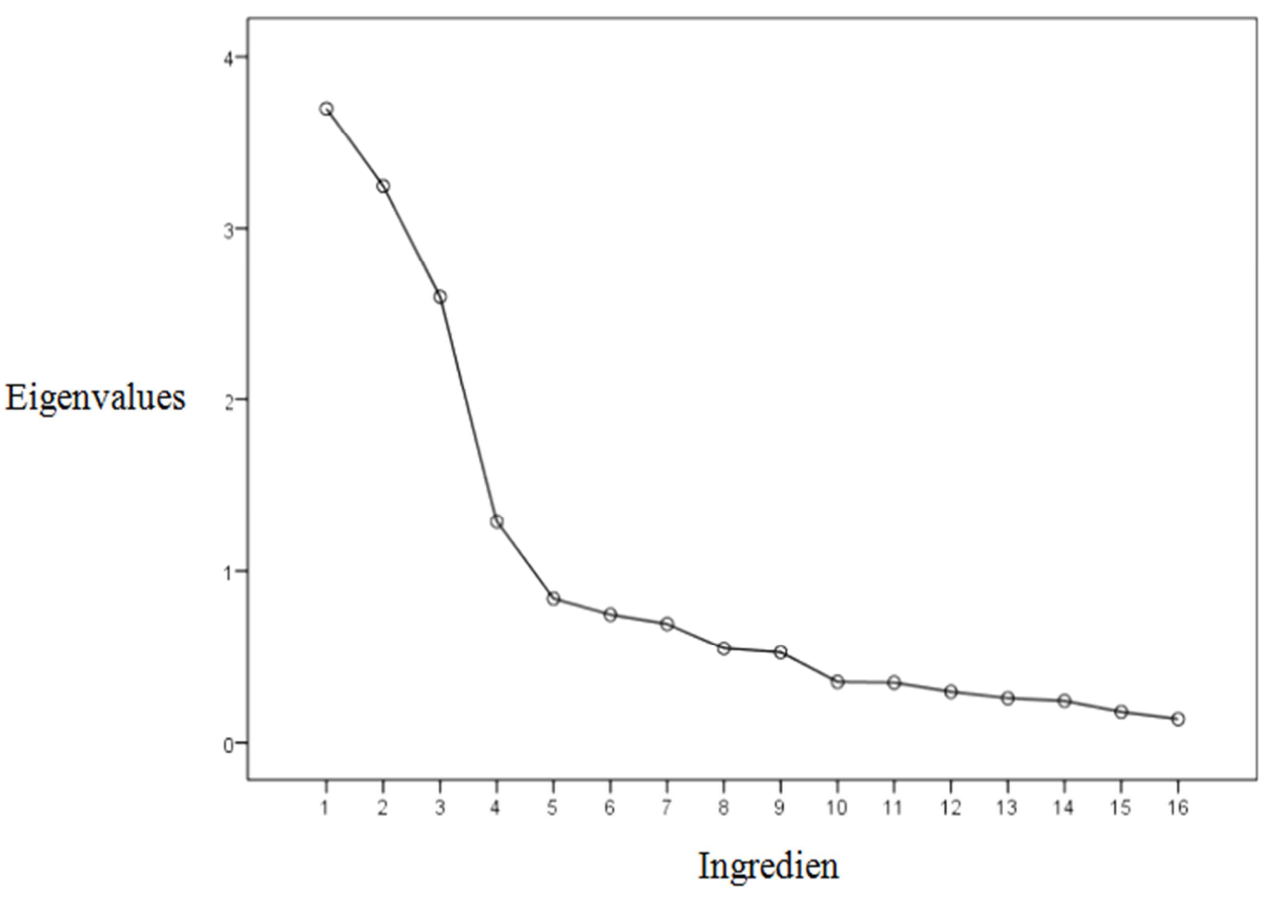

Figure 1. Gravel map.

\subsubsection{Reliability Analysis}

Result of the Retest reliability are shown in Table 6. Result of the Cronbach's $\alpha$ coefficient are shown in Table 7.

Table 6. Result of the Retest reliability.

\begin{tabular}{llll}
\hline Dimension & First Score & Second Score & Pearson Correlation Coefficient \\
\hline Special dietary knowledge & $24.23 \pm 7.78$ & $24.37 \pm 7.17$ & 0.990 \\
Healthy dietary attitude & $18.50 \pm 1.82$ & $18.37 \pm 1.56$ & 0.893 \\
Dietary behavior & $18.37 \pm 3.80$ & $18.33 \pm 2.91$ & 0.916 \\
Risk prevention & $8.23 \pm 2.19$ & $8.27 \pm 2.10$ & 0.997 \\
Total Scale & $71.92 \pm 9.31$ & $69.33 \pm 9.28$ & 0.975 \\
\hline
\end{tabular}

Table 7. Result of the Cronbach's a coefficient.

\begin{tabular}{lll}
\hline Dimension & Number of items & Cronbach's $\boldsymbol{\alpha}$ coefficient \\
\hline Special dietary knowledge & 5 & 0.756 \\
Healthy dietary attitude & 4 & 0.884 \\
\hline
\end{tabular}




\begin{tabular}{lll}
\hline Dimension & Number of items & Cronbach's $\boldsymbol{\alpha}$ coefficient \\
\hline Dimension 3 & 2 & 0.746 \\
Dietary behavior & 5 & 0.822 \\
Total Scale & 16 & 0.713 \\
\hline
\end{tabular}

\subsubsection{Validity Analysis}

The total content validity index (S-CVI) is 0.981 , and the content validity index (I-CVI) of each item is between 0.913 and 1.0. The results of confirmatory factor analysis showed that the model was slightly modified by the correlation of the residual terms. The correct ed structural equation model is shown in Figure 2. Except for $\mathrm{NFI}=0.769<0.9$, $\mathrm{CMIN} / \mathrm{DF}=1.191, \mathrm{RMSEA}=0.053, \mathrm{PNFI}=0.628, \mathrm{TLI}=0.940$. The fitting indexes such as IFI $=0.954$ and $\mathrm{CFI}=0.951$ are all within the reference range, and the model has a good matching degree with the scale (Figure 2).

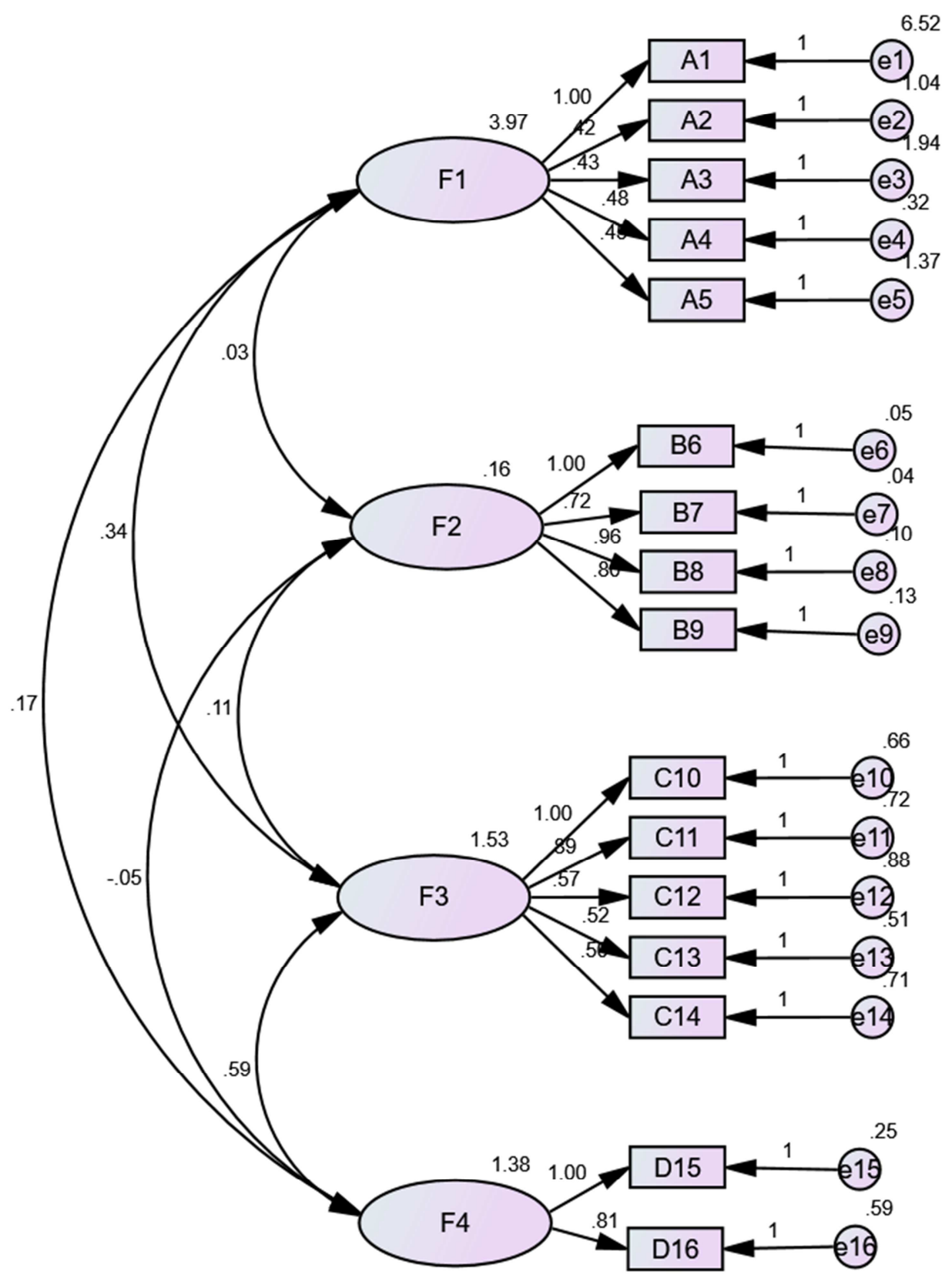

Figure 2. Verification factor analysis structural equation model diagram.

\section{Discussion}

With the continuous advancement of molecular diagnostics, more and more patients with Citrin deficiency have been diagnosed [14-16]. At present, the clinical research on this disease mostly focuses on molecular diagnosis, clinical manifestations and treatment. Children as a special group lack 
self-management ability, and their management ability depends on the caregiver, but there is currently no clinical scale to evaluate the caregiver's ability to manage the diet of children with citrin deficiency.

\subsection{Rationality Analysis of Scale Dimensions and Items}

There are six steps in the construction of the scale, which are to clarify the purpose of measurement, assign dimensions and weights, establish the original pool of entries, select expression of the entries, pre-experiment and analysis, revision and improvement [13]. This study is guided by the above steps to ensure the rigor of the scale development. In this study, an expert correspondence form consisting of 3 dimensions and 31 items was formed through literature review, expert consultation in related fields, and group discussions. The dimensions and items of the scale were modified through two rounds of Delphi expert correspondence. The caregivers of children with Citron deficiency disease tested the expression of the items, and finally formed an initial scale of 3 dimensions and 31 items.

\subsection{Reliability Analysis of Expert Consultation}

The experts invited in this study are from 10 provinces, autonomous regions, and municipalities directly under the Central Government in China. The institutions represented are all tertiary hospitals or colleges and universities. In addition to one professional nursing specialist, the rest of the experts held titles of deputy senior and above. They all had profound knowledge and rich experience in clinical nursing, clinical nutrition and hereditary liver disease ensuring the representativeness and authority of the Delphi expert consultants.

The recovery rates of the two questionnaires were $100 \%$ and $95.8 \%$, and the expert opinion submission rates were $58.3 \%$ and $52.2 \%$ in this study. The results show that the experts participating in the survey were highly motivated and the consultation results are reliable. With the expert authority coefficient $(\mathrm{Cr}) \geq 0.70$, the consultation result is authoritative [10]. The Cr values of the two rounds of consultation in this study were 0.900 and 0.887 meaning there was a high degree of expert authority.

The coefficient of variation (vj) and Kendall's W are used to indicate the degree of coordination of expert opinions, $\mathrm{vj}<$ 0.25 , and Kendall's W value is $0-11$ [17]. The dimensions of Kendall's W are 0.187 and 0.201 , the Kendall's W of the items are 0.212 and $0.30(P<0.05)$ in the two surveys of this study. The level of expert coordination maintained an upward trend in this study.

\subsection{Analysis of the Screening Process for Items}

Screening was performed again by item analysis, Cronbach's a coefficient method, and exploratory factor analysis in this study.

The item analysis method is to test the difference between the scores of the high- and low-level survey respondents on each item. In order to evaluate whether the items have good discrimination and $27 \%$ is usually used as the cutoff value [13]. All items meet the screening criteria in this survey $(\mathrm{CR}>3.0, P$ $<0.001$ ) meaning that all items had a high degree of discrimination for survey subjects at different levels, and no items were deleted by this method.

Cronbach's $\alpha$ method performs item screening from the perspective of internal consistency. The principle of this method is that if an entry is deleted, Cronbach's $\alpha$ is rises, which means that the item will reduce the internal consistency of the scale, and this entry should be deleted. The researchers deleted the items A3, A8, B13, C14, C18, C19, and C21, to assure the internal consistency of the scale.

Exploratory factor analysis screens items from a representative perspective. If an item has a large load on a common factor, that item can reflect the characteristics of the dimension represented by the common factor, but if an item has a large load on two or more common factors, the attribute of the entry is not strong and the representation is poor. Exploratory factor analysis was repeated 7 times, and 4 common factors were extracted. Finally, the dimensions of the scale were re-divided.

\subsection{Evaluation}

The feasibility of the scale is expressed by the recovery rate and efficiency. Usually, the effective recovery rate should be $\geq 85 \%$, and the effective recovery rate is $86.6 \%$ in this survey, and means the scale has high acceptability. Cronbach's $\alpha$ is an index used to evaluate the degree of internal consistency of the scale, and it is required to be $>0.7$. Cronbach's $\alpha$ in each dimension is $0.786,0.733,0.871,0.779$ in this study, and indicates that the internal consistency of this scale is high. The retest reliability coefficient of the scale is 0.975 , which shows good external stability. The total content validity index (S-CVI) is 0.981 , and the content validity index (I-CVI) of each item is between 0.913 and 1 , which showed the high content validity in this study. Confirmatory factor analysis was used for structural inspection of the scale. The researchers slightly modified the model, and the fitting indicators such as IFI and CFI were within the reference range, indicating that the model matches the scale well and the scale results are good.

\section{Conclusions}

The first draft of the citrin deficiency disease diet management scale was formed through two rounds of the Delphi method, and consisted of 3 dimensions and 31 items. The final scale was formed by re-screening all items through classical measurement theory, which ultimately consisted of 4 dimensions and 16 items. Screening methods included item analysis, Cronbach's $\alpha$ coefficient method, and factor analysis. All analyses indicated the scale had high reliability and validity for determining the caregivers, of children with $\mathrm{CD}$, attitudes, knowledge and behaviors concerning dietary practices. The scale can be confidently used to evaluate the caregiver's ability to manage a child's dietary needs when dealing with $\mathrm{CD}$. 


\section{References}

[1] Kobayashi K, Sinasac D, Iijima M, et al. The gene mutated in adult-onset type II citrullinaemia encodes a putative mitochondrial carrier protein [J]. Nature Genetics, 1999, 22 (2): 159-163.

[2] Song Y Z, Deng M, Chen F P, et al. Genotypic and phenotypic features of citrin deficiency: Five-year experience in a Chinese pediatric center [J]. International Journal of Molecular Medicine, 2011, 28 (1): 33-40.

[3] Fukushima K, Yazaki M, Nakamura M, et al. Conventional Diet Therapy for Hyperammonemia is Risky in the Treatment of Hepatic Encephalopathy Associated with Citrin Deficiency [J]. Internal Medicine, 2010, 49 (3): 243-247.

[4] Imamura Y, Kobayashi K, Shibatou T, et al. Effectiveness of carbohydrate-restricted diet and arginine granules therapy for adult-onset type II citrullinemia: a case report of siblings showing homozygous SLC25A13 mutation with and without the disease [J]. Hepatology Research, 2003, 26 (1): 68-72.

[5] Kimura N, Kubo N, Narumi S et al. Liver Transplantation Versus Conservative Treatment for Adult-Onset Type II Citrullinemia: Our Experience and a Review of the Literature [J]. Transplantation Proceedings, 2013, 45 (9): 3432-3437.

[6] Hayasaka K, Numakura C, Toyota K, et al. Medium-chain triglyceride supplementation under a low-carbohydrate formula is a promising therapy for adult-onset type II citrullinemia [J]. Molecular Genetics and Metabolism Reports, 2014, 1: 42-50.

[7] Saheki T, Kobayashi K, Terashi M, et al. Reduced carbohydrate intake in citrin-deficient subjects [J]. Journal of Inherited Metabolic Disease, 2008, 31 (3): 386-394.

[8] Hayasaka K, Numakura C, Yamka M, et al. Medium-chain triglycerides supplement therapy with a low-carbohydrate formula can supply energy and enhance ammonia detoxification in the hepatocytes of patients with adult-onset type II citrullinemia [J]. Journal of Inherited Metabolic Disease, 2018, 41 (5): 777-784.

[9] Numakura C, Tamiya G, Ueki M, et al. Growth impairment in individuals with citrin deficiency $[\mathrm{J}]$. Journal of Inherited Metabolic Disease, 2019, 42 (3): 501-508.

[10] Ge G. Development and evaluation of pre-diabetes self-management scale, [D]. Jinan University, 2016.

[11] Huang M, Gu Y, Zhang Y X, et al. Development and Evaluation of Evidence and Validity of Evidence-based Nursing Practice Readiness Assessment Scale [J]. Chinese Journal of Evidence-based Pediatr, 2017, 12 (2): 121-125.

[12] Rong Y M, Zhang Y X, Lou J H, et al. Development of patient care and family-centered nursing process scale and its reliability and validity evaluation [J]. Chinese Journal of Evidence-based Pediatr, 2015, 10 (2): 136-141.

[13] $\mathrm{Wu} \mathrm{M} \mathrm{L.} \mathrm{Questionnaire} \mathrm{statistical} \mathrm{analysis} \mathrm{practice-SPSS}$ operation and application [M]. Chongqing University Press, 2010: $159-160$.

[14] Song Y Z, Zhang Z H, Lin W X, et al. SLC25A13 gene analysis in citrin deficiency: sixteen novel mutations in East Asian patients, and the mutation distribution in a large pediatric cohort in China [J]. PloS ONE, 2013, 8 (9): e74544.

[15] Zhang Z H, Yang ZG, Chen F P, et al. Screening for five prevalent mutations of SLC25A13 gene in Guangdong, China: a molecular epidemiologic survey of citrin deficiency [J]. The Tohoku Journal of Experimental Medicine, 2014, 233 (4): 275-281.

[16] Xu J, Gao M. Analysis of SLC25A13 gene mutations in five infants with neonatal intrahepatic cholestasis caused by citrin deficiency [J]. Chinese journal of medical genetics, 2018, 35 (1): 34-38.

[17] Zeng G. Modern epidemiological methods and application [M]. Beijing Medical University/China Union Medical University Joint Press, 1994: 250-270. 